Research paper

\title{
Molecular epidemiology and genetic diversity of norovirus infection in children hospitalized with acute gastroenteritis in East Java, Indonesia in 2015-2019
}

\author{
Takako Utsumi $^{\text {a,b }}$, Maria Inge Lusida ${ }^{\text {a }}$, Zayyin Dinana ${ }^{\text {a }}$, Rury Mega Wahyuni ${ }^{\text {a }}$, \\ Soegeng Soegijanto ${ }^{\mathrm{a}}$, Soetjipto ${ }^{\mathrm{a}}$, Alpha Fardah Athiyyah ${ }^{\mathrm{a}, \mathrm{c}}$, Subijanto Marto Sudarmo ${ }^{\mathrm{a}, \mathrm{c}}$, \\ Reza Gunadi Ranuh ${ }^{\text {a,c }}$, Andy Darma ${ }^{\text {a,c }}$, Juniastuti ${ }^{a}$, Laura Navika Yamani ${ }^{a}$, Yen Hai Doan ${ }^{\text {d,e }}$, \\ Hiroyuki Shimizu $^{\mathrm{d}}$, Koji Ishii ${ }^{\mathrm{f}}$, Chieko Matsui ${ }^{\mathrm{b}}$, Lin Deng ${ }^{\mathrm{b}}$, Takayuki Abe ${ }^{\mathrm{b}}$, \\ Kazuhiko Katayama ${ }^{g}$, Ikuo Shoji ${ }^{\text {b, }}$ \\ ${ }^{a}$ Indonesia-Japan Collaborative Research Center for Emerging and Re-emerging Infectious Diseases, Institute of Tropical Disease, Airlangga University, Surabaya, \\ Indonesia \\ ${ }^{\mathrm{b}}$ Center for Infectious Diseases, Kobe University Graduate School of Medicine, Kobe, Japan \\ ${ }^{\mathrm{c}}$ Department of Child Health, Soetomo Hospital, Airlangga University, Surabaya, Indonesia \\ ${ }^{\mathrm{d}}$ Department of Virology II, National Institute of Infectious Diseases, Tokyo, Japan \\ ${ }^{\mathrm{e}}$ Department of Environmental Parasitology, Tokyo Medical and Dental University, Japan \\ ${ }^{\mathrm{f}}$ Department of Quality Assurance and Radiological Protection, National Institute of Infectious Diseases, Tokyo, Japan \\ ${ }^{\mathrm{g}}$ Laboratory of Viral Infection 1, Department of Infection Control and Immunology, Ömura Satoshi Memorial Institute and Graduate School of Infection Control Sciences, \\ Kitasato University, Tokyo, Japan
}

\section{A R T I C L E I N F O}

\section{Keywords:}

Norovirus

RdRp/capsid genotype

Epidemiology

Children

Indonesia

\begin{abstract}
A B S T R A C T
Noroviruses are recognized as a leading cause of outbreaks and sporadic cases of acute gastroenteritis (AGE) among individuals of all ages worldwide, especially in children $<5$ years old. We investigated the epidemiology of noroviruses among hospitalized children at two hospitals in East Java, Indonesia. Stool samples were collected from 966 children with AGE during September 2015-July 2019. All samples were analyzed by reverse transcription-polymerase chain reaction (RT-PCR) for the amplification of both the RNA-dependent RNA polymerase (RdRp) and the capsid genes of noroviruses. The genotypes were determined by phylogenetic analyses. In 2015-2019, noroviruses were detected in 12.3\% (119/966) of the samples. Children <2 years old showed a significantly higher prevalence than those $\geq 2$ years old $(P=0.01)$. NoV infections were observed throughout the year, with the highest prevalence in December. Based on our genetic analyses of RdRp, GII. [P31] (43.7\%, 31/71) was the most prevalent RdRp genotype, followed by GII.[P16] (36.6\%, 26/71). GII.[P31] was a dominant genotype in 2016 and 2018, whereas GII.[P16] was a dominant genotype in 2015 and 2017. Among the capsid genotypes, the most predominant norovirus genotype from 2015 to 2018 was GII.4 Sydney_2012 (33.6\%, 40/ 119). The most prevalent genotype in each year was GII.13 in 2015, GII.4 Sydney_2012 in 2016 and 2018 , and GII.3 in 2017. Based on the genetic analyses of RdRp and capsid sequences, the strains were clustered into 13 RdRp/capsid genotypes; 12 of them were discordant, e.g., GII.4 Sydney[P31], GII.3[P16], and GII.13[P16]. The predominant genotype in each year was GII.13[P16] in 2015, GII.4 Sydney[P31] in 2016, GII.3[P16] in 2017, and GII.4 Sydney[P31] in 2018. Our results demonstrate high detection rates and genetic diversity of norovirus GII genotypes in pediatric AGE samples from Indonesia. These findings strengthen the importance of the continuous molecular surveillance of emerging norovirus strains.
\end{abstract}

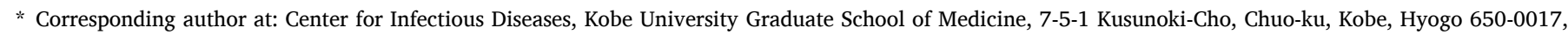
Japan

E-mail address: ishoji@med.kobe-u.ac.jp (I. Shoji).
} 


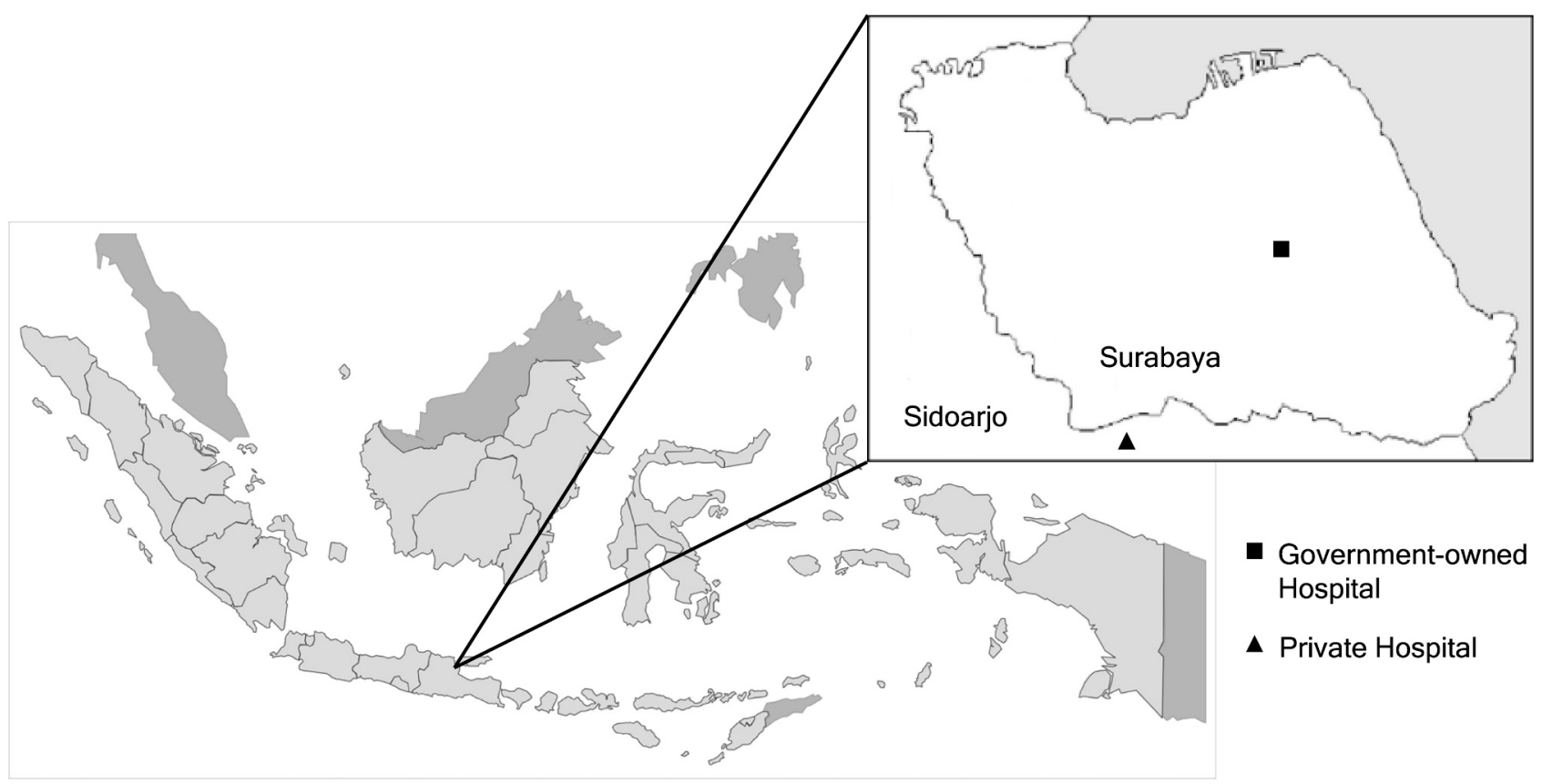

Fig. 1. Geographic location of two hospitals in this study where stool samples were collected in 2015-2019.

\section{Introduction}

Noroviruses are recognized as a leading cause of outbreaks and sporadic cases of acute gastroenteritis (AGE) among people of all age groups worldwide (Bon et al., 2005; Khamrin et al., 2016; Zheng et al., 2010). Epidemiological studies estimated that worldwide, noroviruses may cause $>1$ million hospitalizations and up to 200,000 deaths annually in children $<5$ years old (Ahmed et al., 2014; Bányai et al., 2018). In Indonesia, norovirus gastroenteritis occurs sporadically in children with a prevalence of 15 to $61 \%$, and hospitalization is often required for the patients (Hakim et al., 2018; Nirwati et al., 2019; Athiyyah et al., 2020; Wulandari et al., 2020).

Noroviruses are a positive-sense, single-stranded, non-enveloped RNA viruses that belong to the family Caliciviridae (Jiang et al., 1993). It has been suggested that the norovirus genome can evolve rapidly, resulting in many different genotypes (Kobayashi et al., 2016). The noroviruses are currently classified into 10 genogroups (GI to GX) based on the sequence diversity in ORF2 encoding the VP1 protein (Parra, 2019). Only genogroups GI, GII, and GIV are known to infect humans. These genogroups are further divided into multiple genotypes (nine GI genotypes, 27 GII genotypes, and two GIV genotypes) (Chhabra et al., 2019; Vinjé, 2015). Because recombination of the norovirus genome frequently occurs at the ORF1-ORF2 intersect, resulting in different capsid and polymerase gene genotypes, the current nomenclature comprises both polymerase (RdRp) and capsid genotypes (VP1).

GI and GII viruses are currently classified into approx. 14 and $37 \mathrm{P}-$ genotypes, respectively on the basis of their RdRp gene sequences (Chhabra et al., 2019). Although noroviruses are antigenically and genetically diverse, a single genotype, i.e., GII.4, has been the predominant genotype globally. This genotype has been associated with not only sporadic gastroenteritis in children but also outbreaks in adults in various countries (Motoya et al., 2019; Wu et al., 2015; Polkowska et al., 2014; Medici et al., 2009).

It was reported that the norovirus prevalence among hospitalized children was $18.5 \%$, and that the most prevalent genotype in 2015 in Yogyakarta/West Nusa Tenggara, Indonesia was GII.4 Sydney[P31] (56\%) (Nirwati et al., 2019). There has been no study of the transition of norovirus genotypes circulating in Indonesia using a long-term perspective. We thus conducted the present study to investigate the transition of genotypes of norovirus among children in East Java, Indonesia, during the years 2015-2019.

\section{Materials and methods}

\subsection{Stool sample collection}

A total of 966 stool samples were collected from pediatric patients who were admitted to two hospitals in East Java, Indonesia with a clinical diagnosis of AGE during the period from June 2015 to July 2019. Four-hundred seventy-five samples $(49.2 \%)$ were collected from a private hospital in a suburb of the city of Surabaya, and the other 491 samples $(50.8 \%)$ were from a government-owned hospital in the same city (Fig. 1). The ages of the patients ranged from 1 to 191 months (median, 12 months), and $89.5 \%$ of the total patients were $<5$ years old. Of the 966 patients, $572(59.2 \%)$ were male and 394 (40.8\%) were female. AGE was defined as $\geq 3$ episodes of watery or looser-than normal stools per day, lasting $<14$ days. Written informed consent for the patients' materials to be used was obtained from the parents of all of the children. Demographic data (age, sex) and clinical data were also recorded. The study protocol was reviewed and approved by the Ethics Committees of Airlangga University, the two hospitals in Indonesia, and Kobe University in Japan. Stool samples were collected and stored at $-20{ }^{\circ} \mathrm{C}$ at the hospitals until they were transported to the Institute of Tropical Disease, Airlangga University in a frozen condition.

\subsection{Norovirus detection}

A $10 \%(w / v)$ stool suspension of each sample in distilled water was prepared by centrifugation at $20,000 \mathrm{xg}$ for $10 \mathrm{~min}$. Viral RNA was extracted from $140 \mu$ l of supernatant with a QIAamp Viral RNA Mini Kit (Qiagen, Hilden, Germany) following the manufacturer's instructions. The RNA was eluted with $60 \mu \mathrm{l}$ of RNase-, DNase-free water and stored at $-80^{\circ} \mathrm{C}$. The reverse transcription (RT) reaction for the capsid gene and polymerase gene was performed at $65^{\circ} \mathrm{C}$ for $3 \mathrm{~min}$, followed by $40^{\circ} \mathrm{C}$ for $1 \mathrm{~h}$ using Superscript III reverse transcriptase (Invitrogen, New York, US) and random primers (Takara Bio, Kyoto, Japan).

Genogroup-specific primers and probes were used to detect noroviruses by TaqMan-based quantitative real-time polymerase chain 
reaction (PCR) using an ABI 7300 real-time PCR system (Applied Biosystems, Foster City, CA) (Utsumi et al., 2017). The primer and probe sets (COG1F/COG1R/RING1-TP(a)/RING1-TP(b)) and (COG2F/ COG2R/ALPF/RING2AL-TP) were used to screen for GI and GII norovirus strains, respectively. Real-time PCR for GI and GII were performed separately, and negative and positive controls were included in each experiment. The amplification was performed as described (Kageyama et al., 2003; Khamrin et al., 2016).

One hundred-nineteen norovirus-positive samples were determined by real-time PCR. We then performed a conventional RT-PCR to confirm the norovirus RNA and sequencing. The primer pairs of G1SKF/G1SKR and G2SKF/G2SKR were used for the detection of the capsid gene for norovirus GI and GII, respectively (Kojima et al., 2002). To investigate the presence of recombination of the polymerase gene for norovirus GII, we used the primer pairs of $1 \mathrm{~S} / 1 \mathrm{~A}$ for the 1st PCR and 2S/2A for the 2nd PCR (Utsumi et al., 2017), following the described amplification conditions (Kojima et al., 2002). RT-PCR for norovirus GI in the polymerase gene was not performed in this study, since GI strains were quite rare $(3.4 \%)$.

\subsection{Sequencing and phylogenetic analyses}

The sequences of the 119 norovirus amplicons in the capsid gene and 71 amplicons in the polymerase gene were determined directly from the PCR product which were successfully amplified with the BigDye terminator cycle sequencing kit and 3500XL genetic analyzer (Applied Biosystems). Therefore, both the capsid and the RdRp genes for 71 samples were analyzed and the remaining capsid genes for 48 samples were analyzed. Phylogenetic analyses were performed based on the approx. 270-bp nucleic acid sequences of the amplified norovirus capsid region and the 380-bp nucleic acid sequences of the polymerase region. To create norovirus GII phylogenetic trees of the capsid gene and polymerase gene, we analyzed representative strains. Reference sequences were retrieved from the DNA Data Bank of Japan/European Molecular Biology Laboratory/GenBank database. Alignments were performed using CLUSTAL X software (ver. 1.83), and the phylogenetic trees were constructed by the neighbor-joining method based on the Tamura-Nei substitution model. To confirm the reliability of the phylogenetic tree analysis, bootstrap resampling and reconstruction were carried out 1000 times. These analyses were carried out using Molecular Evolutionary Genetic Analysis (MEGA4) software (Tamura et al., 2007). The recombination point of norovirus strains were determined with SimPlot software v.3.5.1 (Lole et al., 1999). The bootscan and maximum chi-square (MaxChi) test using the Recombination Detection Program v.4.101 (RDP4) was used and confirmed with significant events $(p<0.01)$ (Martin et al., 2015).

The genetic sequences described herein have been deposited in the GenBank database under accession numbers (LC576043- LC576207).

\subsection{Statistical analyses}

The statistical analyses were performed by the chi-squared test or Fisher's exact test for categorical variables using SPSS Statistics 17.0 (Advanced Analytics, Tokyo). Results were considered significant at $P<$ 0.05 .

\section{Results}

\subsection{The prevalence of norovirus}

A total of 119 (12.3\%) samples of the 966 collected from pediatric patients during the study period were norovirus-positive in the capsid region as shown by RT-PCR. The prevalence of norovirus infection was significantly higher at the government-owned hospital (14.7\%) compared to the private hospital $(9.9 \%)(P=0.024)$. The prevalence of norovirus infection ranged from $17.4 \%$ in 2015 to $10.3 \%$ in 2018

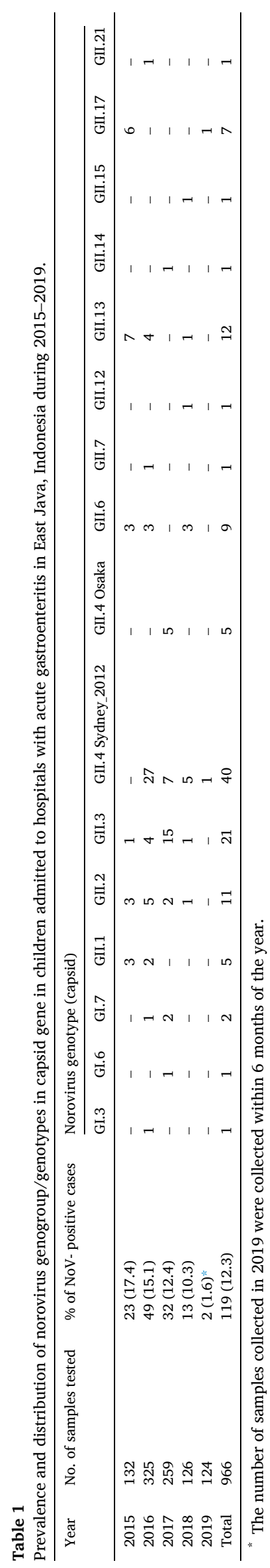


Table 2

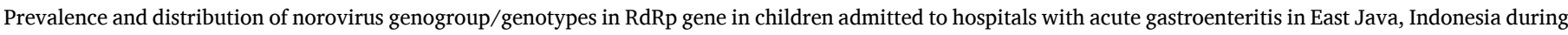
2015-2019.

\begin{tabular}{|c|c|c|c|c|c|c|c|c|c|}
\hline \multirow[t]{2}{*}{ Year } & \multirow[t]{2}{*}{ No. of samples tested } & \multirow[t]{2}{*}{$\%$ of norovirus- positive cases } & \multicolumn{7}{|c|}{ Norovirus genotype (RdRp) } \\
\hline & & & GII.P7 & $\mathrm{GII} \cdot \mathrm{P} 12$ & GII·P15 & GII.P16 & GII.P17 & GII·P31 & GII.P33 \\
\hline 2015 & 132 & $12(9.1)$ & 4 & - & - & 5 & 2 & - & 1 \\
\hline 2016 & 325 & $32(9.8)$ & 1 & 2 & - & 5 & 1 & 23 & - \\
\hline 2017 & 259 & $18(6.9)$ & 1 & - & - & 13 & - & 4 & - \\
\hline 2018 & 126 & $8(6.3)$ & 1 & - & 1 & 3 & - & 3 & - \\
\hline 2019 & 124 & $1(0.8)^{*}$ & - & - & - & - & - & 1 & - \\
\hline Total & 966 & $71(7.3)$ & 7 & 2 & 1 & 26 & 3 & 31 & 1 \\
\hline
\end{tabular}

* The number of samples collected in 2019 were collected within 6 months of the year.

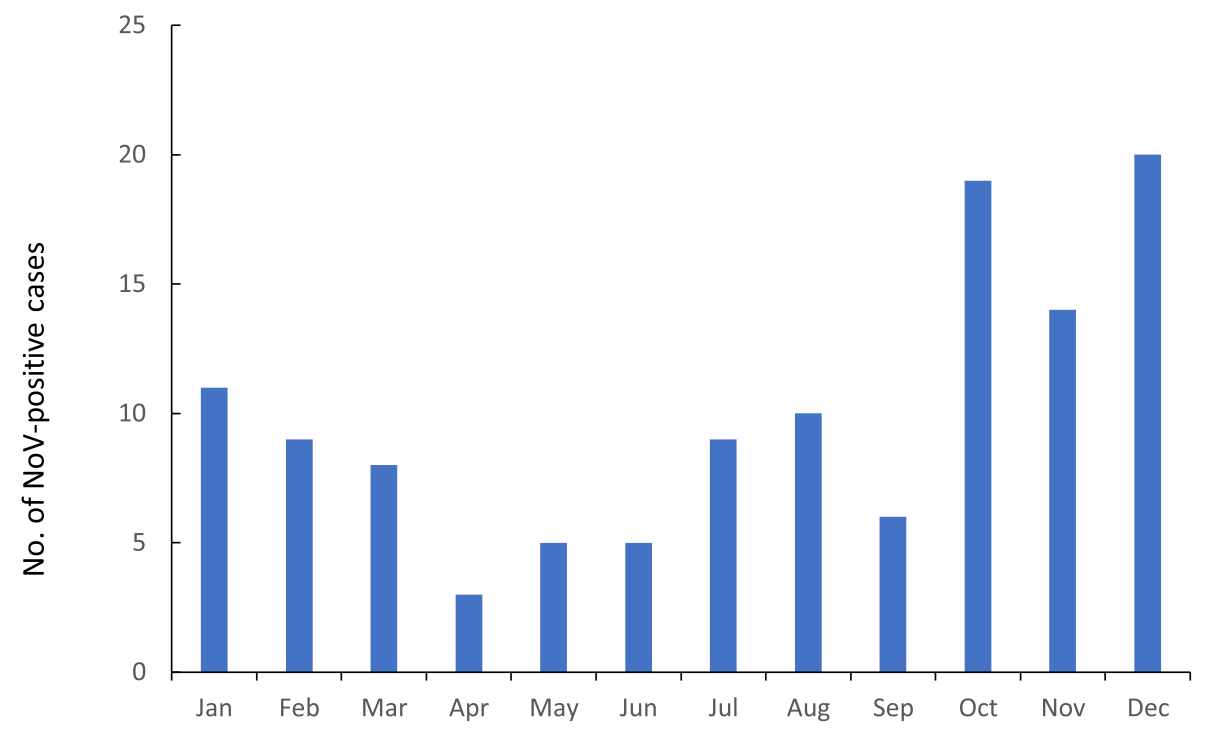

Months in 2015-2019

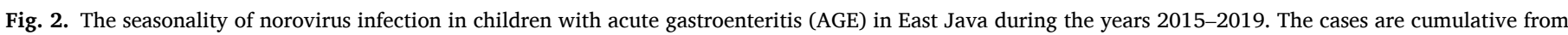
2015 to 2019. In Indonesia, there are two seasons: the rainy season (November-April) and the dry season (May-October).

(Tables 1 and 2). The seasonality of norovirus infection in the children with AGE during 2015-2019 is illustrated in Fig. 2. Norovirus infections were detected throughout the year with the peaks in December (rainy season), followed by October (early rainy season), and November (rainy season). Approximately $85 \%$ (101/119) of the norovirus-positive samples were detected in children $<2$ years old, showing significantly higher prevalence than those $\geq 2$ years old $(P=0.01)$. The median age of the norovirus-positive children was 12 months (range 1-96 months), and $58.8 \%(70 / 119)$ of the norovirus-positive children were male.

\subsection{Norovirus genotypes in capsid gene}

Of the 119 norovirus-positive samples in the capsid gene, 116 (97.5\%) were norovirus GII and four (3.4\%) were norovirus GI. There was one mixed infection of norovirus GI and GII (0.8\%). The capsid genes of the 119 norovirus strains were successfully sequenced, and the prevalence of genotypes were as follows: GII.4 Sydney_2012 $(n=40$, $33.6 \%)$, GII.3 $(n=21,17.6 \%)$, GII.13 $(n=12,10.1 \%)$, GII.2 $(n=11$, 9.2\%), GII.6 ( $n=9,7.6 \%)$, GII.17 ( $n=7,5.9 \%)$, GII.1 $(n=5,4.2 \%)$, GII.4 Osaka ( $n=5,4.2 \%)$, and GI.7 $(n=2,1.7 \%)$; seven strains were identified as GII.7, GII.12, GII.14, GII.15, GII.21, GI.3, and GI.6, respectively (Table 1). The distinct genotypes found in the governmentowned hospital were GI.6, GI.7, GII.7, GII.14, GII.15 and GII.21, while the distinct genotypes in the private hospital were GI.3 and GII.12. However, each of those genotypes was found in only one patient.

The most predominant genotypes in each year were GII.13 and
GII.17 in 2015, GII.4 Sydney_2012 in 2016, GII.3 in 2017, and GII.4 Sydney_2012 in 2018. The GII.4 Sydney_2012 was detected from 2016 to 2019 (Table 1, Figs. 3A, 4A).

\subsection{Norovirus GII genotypes in RdRp gene}

The RdRp gene of the 71 norovirus GII strains were successfully amplified and sequenced, and the prevalence of genotypes were as follows: GII. P31 ( $n=31,43.7 \%)$, GII. P16 ( $n=26,36.6 \%)$, GII. P7 $(n=7$, 9.9\%), GII. P17 ( $n=3,4.2 \%)$, and GII. P12 ( $n=2,2.8 \%)$; two strains (2.8\%) were identified as GII. P33 and P15, respectively (Table 1). The most predominant genotypes of each year were GII. P16 in 2015, GII. P31 in 2016, GII. P16 in 2017, and GII. P31 in 2018 (Figs. 3B, 4B). The distinct genotypes found in the government-owned hospital were GII. P33 $(n=1)$, GII. P12 $(\mathrm{n}=2)$ and GII. P15 $(\mathrm{n}=1)$, while the distinct genotype in the private hospital was GII. P17 $(n=3)$. The nucleotide sequence identities of the RdRp gene between the GII.P31 strains in this study were $99.6 \%-100 \%$, while those in the previous study from other sites (Yogyakarta and Mataram) of Indonesia were $98.8-100 \%$ (Nirwati et al., 2019). Although each of these two groups tended to have their own cluster, the nucleotide sequence identities between the clusters were as high as $97.3 \%-98.4 \%$. The nucleotide sequence identities of the GII.P16 strains between this study and previous study from Indonesia (Yogyakarta) were 95.8-98.4\%. 
(a)

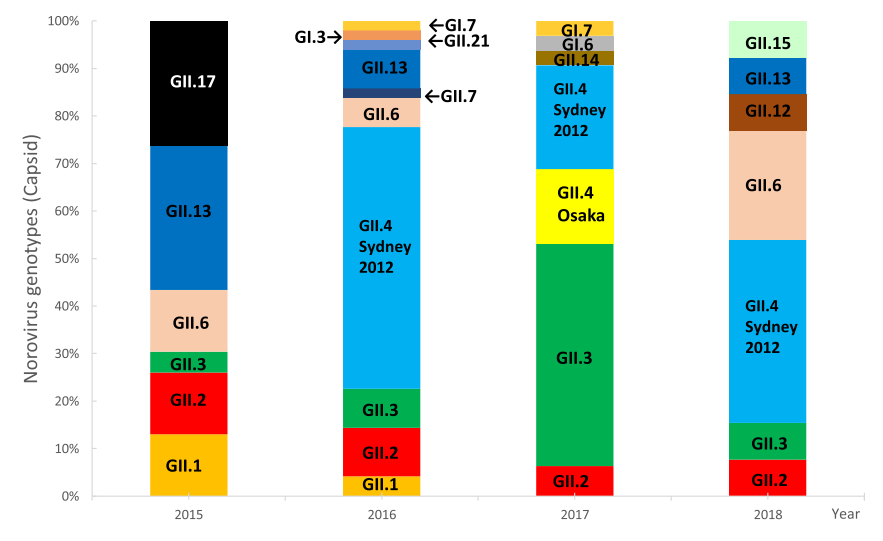

(b)

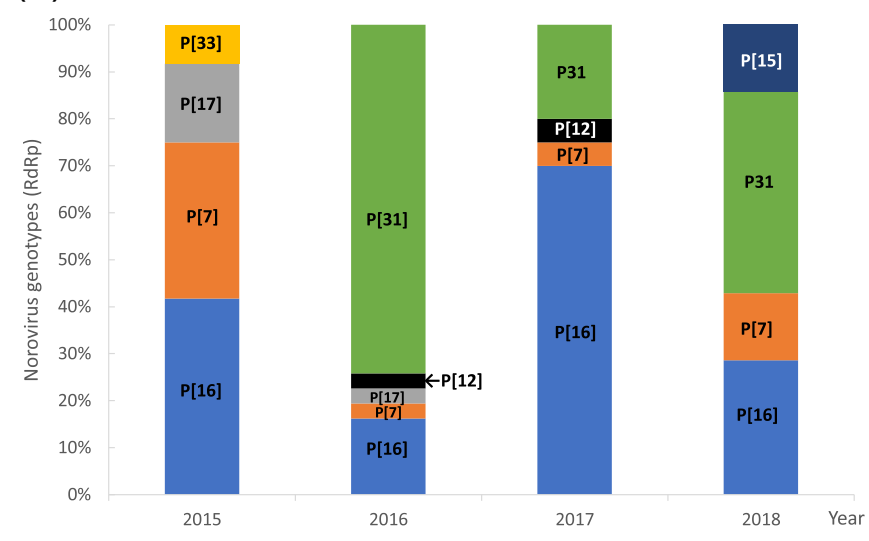

(c)

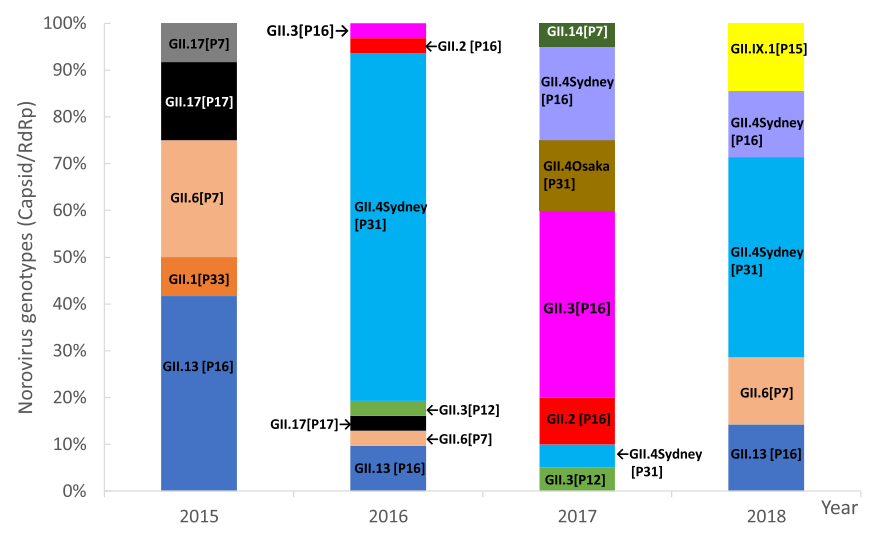

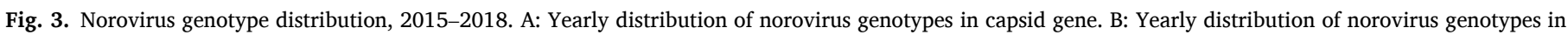
RdRp gene. C: Yearly distribution of norovirus genotypes in RdRp/capsid gene. The data from 2019 are not included because only two cases were available.

\subsection{Combination genotypes of GII noroviruses with RdRp/capsid fragment genes}

Partial nucleotide sequences of the capsid and RdRp genes of norovirus GII were obtained from 71 strains. Based on the 71 capsid and RdRp sequences, the strains were clustered into 13 genotypes, with the following order of prevalence: GII.4 Sydney[P31] $(n=28,39.4 \%)$, GII.13[P16] ( $n=9,12.7 \%)$, GII.3[P16] $(\mathrm{n}=9,12.7 \%)$, GII.4 Sydney [P16] $(n=5,7.0 \%)$, GII.6[P7] $(\mathrm{n}=5,7.0 \%)$, GII.4 Osaka[P31] $(n=3$, 4.2\%), GII.17[P17] ( $\mathrm{n}=3,4.2 \%)$, GII.2[P16] $(\mathrm{n}=3,4.2 \%)$, and GII.3 [P12] ( $n=2,2.8 \%)$; four combination genotypes were identified as GII.1[P33], GII.17[P7], GII.14[P7], and GIX.1[GII.P15], respectively (Fig. 3C). The predominant genotype in this study was GII.4 Sydney [P31]. The predominant genotype each year was GII.13[P16] in 2015, GII.4 Sydney[P31] in 2016, GII.3[P16] in 2017, and GII.4 Sydney[P31] in 2018 (Fig. 3C). The recombinant norovirus strains newly found in Indonesia were GII.4 Sydney[P16] $(n=5)$, GII.3[P12] $(n=2)$, GII.4 Osaka[P31] ( $n=3)$, GII.17[P7] $(n=1)$, and GII.14[P7] $(n=1)$.

Four most common recombinant genotypes (GII.4 Sydney[P31], GII.13[P16], GII.3[P16], GII.4 Sydney[P16]) in this study were analyzed using SimPlot and confirmed the event by RDP4 program (Fig. 5A-D). The ORF1-ORF2 overlap sequences of the four samples indicated the recombination event in the 248-270 nucleotide corresponding the 5067-5089 positions (reference strain: NC-029646) (Fig. 5A-D). The MaxChi and Bootscan results obtained by RDP4 analyses confirm the recombination event. Significant $p$-values were obtained for all the samples used this analyses (Fig. 5A-D).

\section{Discussion}

We performed the present molecular epidemiological study of norovirus infection among children with AGE in East Java, Indonesia whose stools were sampled during the years 2015-2019. Although global outbreaks and sporadic cases of AGE caused by norovirus have been frequently reported, the molecular epidemiology of norovirus in Indonesia has not been well investigated. In addition, no reports about the transition of norovirus prevalence and genogroups/genotypes in Indonesia have been available.

In this study, the overall prevalence of norovirus infection was $12.3 \%$, and the annual prevalence of norovirus infection ranged from $17.4 \%$ in 2015 to $10.3 \%$ in 2018 . A higher prevalence was revealed in the government-owned hospital compared to the private hospital. We speculate that this difference was observed because the governmentowned hospital covers all of East Java province from villages to urban settings, with different hygiene statuses. Rotavirus, another common agent of gastroenteritis, was seen in $281(29.1 \%)$ in this cohort, and 11 (9.2\%) patients were co-infected with norovirus and rotavirus. Although bacterial agents were not searched in this study, high rate of bacterial infection is suspected, since approx. $70 \%$ of pediatric patients were found to be infected with bacteria (Sudarmo et al., 2015).

We also observed that norovirus infection was found extensively in children $<2$ years old, with a significantly higher prevalence compared to that among children $\geq 2$ years old $(P=0.01)$. Although norovirus infection occurred in all age groups, the children $<2$ years old appeared to be more vulnerable, which is consistent with other reports (Wu et al., 2020; Louya et al., 2019; Lu et al., 2019; Siafakas et al., 2018). The low prevalence of norovirus infection among older children could be due to 


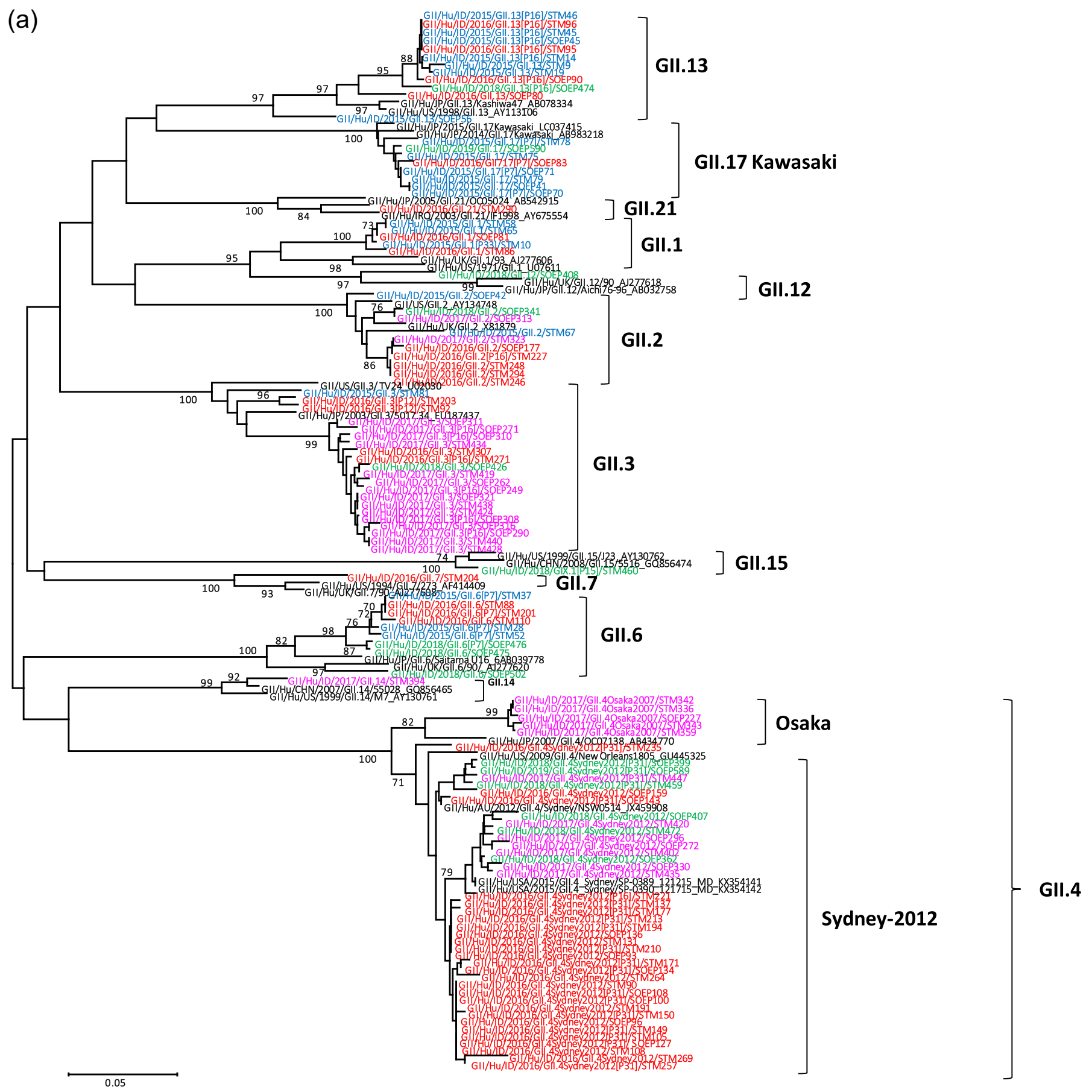

Fig. 4. Phylogenetic analysis of GII norovirus based on the particle nucleotide sequences of the viral capsid (A) and polymerase regions (B). Reference norovirus genotypes are labeled with their GenBank accession numbers. The trees were constructed in MEGA 4 through the neighbor-joining method. The bootstrap values (1000 replicates) are indicated in the phylogenetic tree, and values $<70 \%$ are not shown. The names of the norovirus strains detected in this study are highlighted as follows. 2015: blue, 2016: red, 2017: pink, and 2018-2019: green. Strains sequenced in this study are indicated by a solid circle, the strains sequenced in previous studies in Indonesia are indicated by a solid triangle in Fig. 4B.

acquired immunity through previous exposures (Gupta et al., 2018).

Norovirus GII infections were predominant, representing $97.5 \%$ of the total norovirus infections, which is consistent with studies from other parts of Indonesia and other countries (Athiyyah et al., 2019; Lu et al., 2019; Mathew et al., 2019; Nirwati et al., 2019; Wang et al., 2019). GII noroviruses are more closely related to diseases that require medical treatment, whereas GI noroviruses result in mild symptoms and do not require hospitalization (Siafakas et al., 2018). All of the patients in the present analyses were hospitalized pediatric patients who required medical treatment since they were infected with norovirus GII. However, no clinical differences between genogroups GI and GII were confirmed, because the number of samples detected as GI was too small for a comparison of two genogroups.

Noroviruses are genetically diverse RNA viruses associated with AGE in mammalian hosts (Chhabra et al., 2019). In the present study of noroviruses, we identified three GI genotypes and 13 GII genotypes. The 


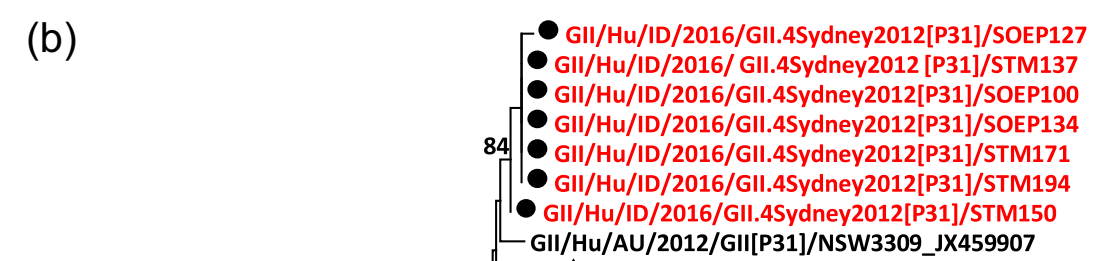

GII/Hu/ID/2016/GII.4Sydney2012[P31]/STM150

GII/Hu/AU/2012/GII[P31]/NSW3309_JX459907

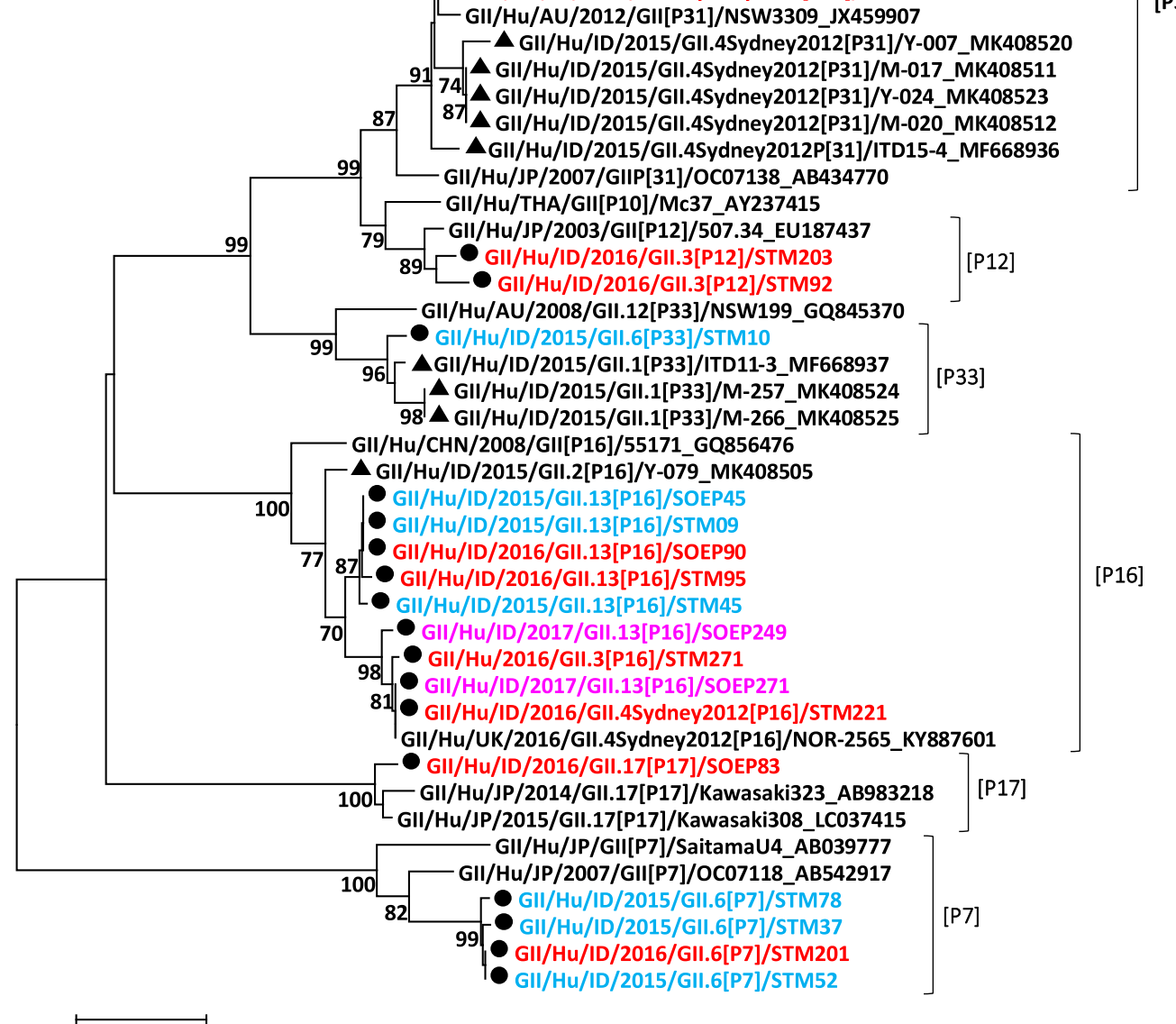

[P31]

0.05

Fig. 4. (continued).

most prevalent genotypes in the capsid gene in 2015 were GII.13 and GII.17, although GII.4 Sydney_2012 was a dominant genotype in other areas of Indonesia (Nirwati et al., 2019). GII.4 noroviruses have been the most common genotype worldwide since 2002 (Cannon et al., 2017). The increase of norovirus infections in pediatric patients from mid-2012 was associated with the emergence of a new variant called Sydney 2012 (Thongprachum et al., 2015). GII.4 Sydney_2012 was predominant in the cohort of present study in 2016 and 2018. In 2013, the predominant norovirus genotype in East Java, Indonesia was GII.4 Sydney_2012 (data not shown). To the best of our knowledge, there are no published data on norovirus genotypes in Indonesia in 2014 or before 2012. The temporal changes of norovirus genotypes are thus poorly understood.

The GII.17 norovirus emerged as a major cause of AGE in the winter of 2014-2015 in several mostly Asian countries, including Japan (Matsushima et al., 2015), Taiwan, China (Jin et al., 2016), and Indonesia, as well as other countries around the world (Giammanco et al., 2017; Matsushima et al., 2019; van Beek et al., 2018). In our present investigation, mainly GII.17 was observed in 2015; it disappeared from 2016 to 2018 and was then detected again in 2019. A rapid replacement of GII.17 by GII.4 in 2016 was reported in other countries (Ao et al., 2017; Liu et al., 2017). Although GII.13 has been considered a minor genotype worldwide (Hoa-Tran et al., 2013), we detected GII.13 as a major genotype in 2015 in this study. GII.13 increased in Nepal during 2005-2011 and in Bhutan during the years 2013-2014 (Hoa-Tran et al., 2015; Wangchuk et al., 2017), which is well consistent with our present findings. The GII.3, GII.2, and GII.6 genotypes observed herein have also been circulating globally as major capsid genotypes.

Our analysis of combined norovirus GII genotypes demonstrated 13 different RdRp/capsid genotypes; 11 of them were distinct RdRp genotypes and capsid genotypes. All of the discordant RdRp/capsid genotypes were suspected to be recombinant strains, and those strains have been reported elsewhere (Chuchaona et al., 2019; Fumian et al., 2016; Liu et al., 2017). GII.13[P16], detected mostly in 2015 in the present study, was isolated in India and European countries at least after 2009 (Hoa-Tran et al., 2015). In our present cohort, the predominant recombinant genotype in 2016 was GII.4 Sydney [P31] (74\%, 23/31) and reported in China and Brazil (Xue et al., 2019; Kuang et al., 2019; Guarines et al., 2020), which is in contrast to the emergence of GII.4 [P16] in the United States in November 2015, and $60 \%$ of all of the norovirus outbreaks in the 2015-2016 season were GII.4 (Cannon et al., 2017).

GII.4 Sydney[P16] first emerged in Indonesia in 2017 and was replaced by GII.4 Sydney[P31] the following year. GII.4[P31] and GII.1 [P33], which were detected in asymptomatic adults in our previous study (Utsumi et al., 2017) were detected among children with AGE in 2015 in the present investigation. Recombinant GII.2[P16] was identified as sporadic cases in Indonesia in September 2016 and in January and November 2017. On the other hand, GII.2[P16] caused AGE outbreaks in October-December 2016 in China (Ao et al., 2017), in Taiwan 


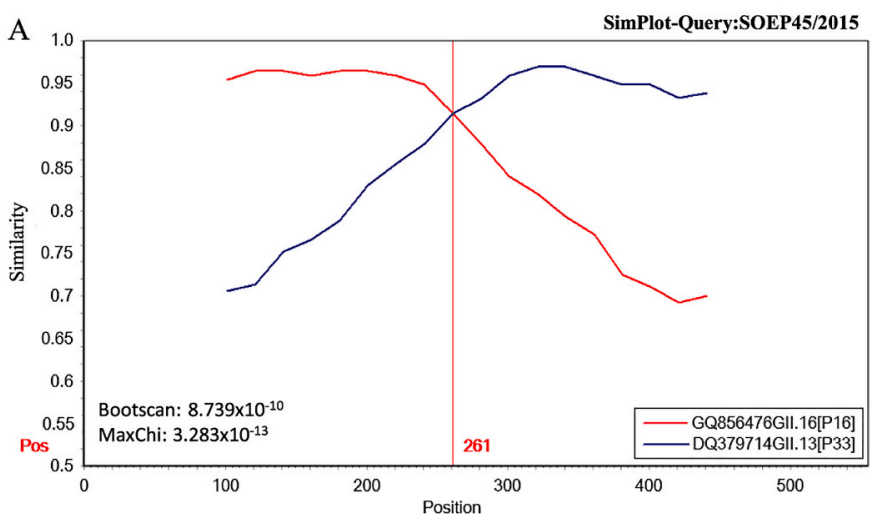

Window. 200 bp, Step: 20 bp, GapStrip: On, Kimura (2-parameter), T/t: 2.0

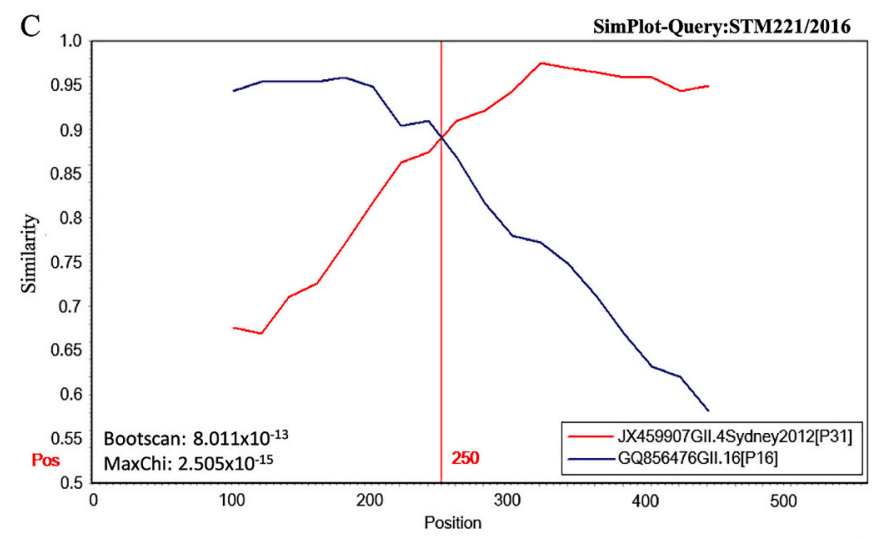

Window: 200 bp, Step: 20 bp, GapStrip: On, Kimura (2-parameter), T/t: 2.0
B

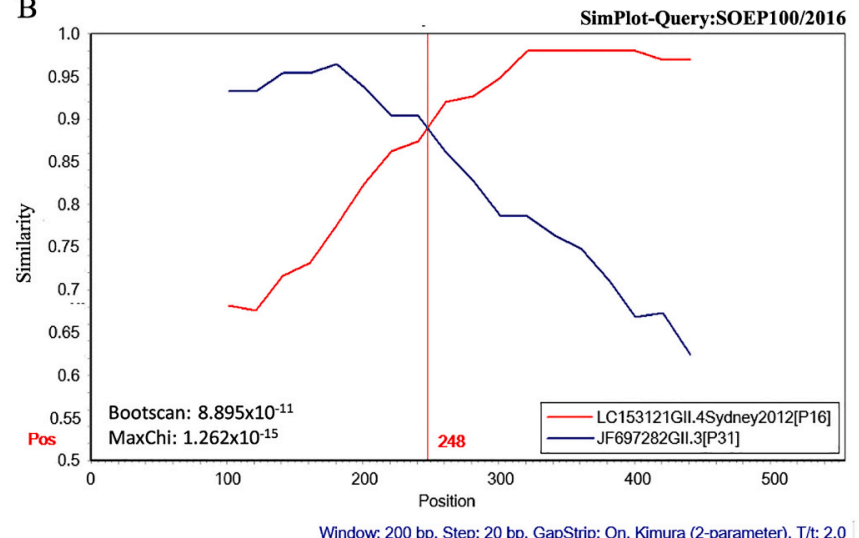

Window: 200 bp, Step: 20 bp, GapStrip: On, Kimura (2-parameter), T/t: 2.0

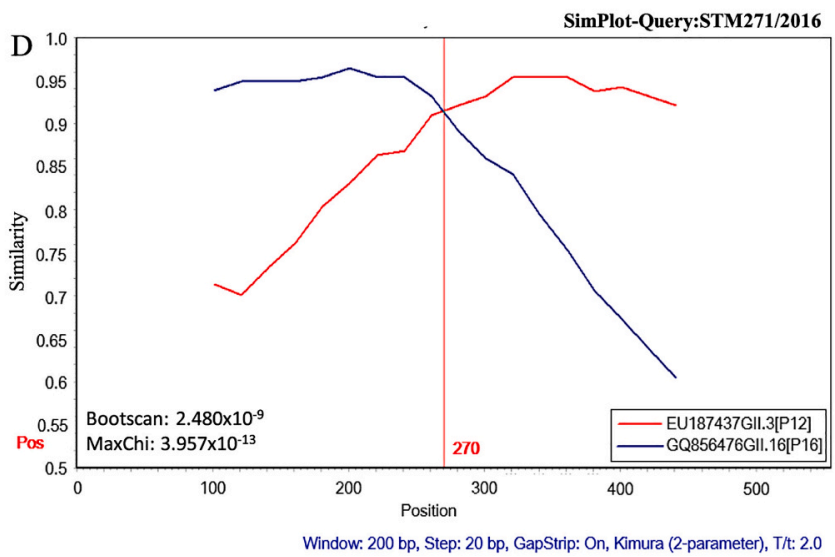

Fig. 5. Simplot analysis of the partial polymerase and capsid gene sequences of the strains GII.4Sydney[P31]/SOEP100 (A), GII.13[P16]/ SOEP45 (B), GII.3[P16] STM271 (C), and GII.4 Sydney[P16]/SOEP221 (D). The assay was performed using standards parameters of the program with a window size of $2000 \mathrm{bp}$, a step size of $20 \mathrm{bp}$ and with the Kimura (2-parameter) model. The query sequences were compared to the reference strains (GII.4 Sydney[P16]/LC153121, GII.3[P31]/JF697282, GII.16[P16]/GQ856476, GII.13[P33]/DQ379714, GII.3[P12]/EU187437, GII.4Sydney[P31]/JX459907). The x-axis indicates the nucleotide positions and y-axis indicates the nucleotide sequence similarity between the recombinant sequence and reference strains. The $p$-values from the Bootscan and MaxChi methods obtained using RDP4 software are shown on the bottom left. The probable nucleotide breakpoint is marked with a red dotted line.

in 2016 (Liu et al., 2017), in Germany in 2016-2017 (Niendorf et al., 2017), and in Japan in 2016 (Nagasawa et al., 2018).

Regarding the RdRp strain P[16], our present analyses revealed that GII.4 Sydney_2012[P16] emerged in 2017 along with GII.2[P16], which is in agreement with a report from France for 2016-2017 (Bidalot et al., 2017) and Yogyakarta in Indonesia in 2015 (Nirwati et al., 2019). GII.4 Sydney [P16] has been detected as a novel recombinant in Germany, the Netherlands, Japan, and China in 2014 and Brazil in 2016 (Barreira et al., 2017; van Beek et al., 2018). GII-13P[16] and GII·3P[16] were detected in Italy over the years 2007-2013 (Medici et al., 2014). In our present study of children with AGE in Indonesia, GII-13P[16] was identified mostly in 2015, 2016 and 2018, and GII.3[P16] was found mostly in 2017. Taken together, our findings indicate that $P[16]$ emerged with multiple capsid genotypes during 2015-2018 in Indonesia. $\mathrm{P}$ [16] was predominant in 2015 and replaced by $\mathrm{P}$ [31] in 2016. This cycle repeats in 2017 (P[16]) and 2018 (P[31]), suggesting that the RdRp region evolves rapidly, similar to the VP1 gene.

GII.4 Osaka[P31] was distinctly detected in 2017 in this study, and it was detected in Japan in 2007, Australia in 2008, and Hong Kong in 2019 (Tse et al., 2020). GII.6[P7], which was identified in outbreaks in Australia in 2012-2014 (Bruggink et al., 2016), was detected in samples from 2015 and 2018 in the present study. GII.4 Sydney 2012[P31] strains circulated in 2016 in this study were closely related to the strains detected in 2015 in other site of Indonesia (Yogyakarta).

The epidemiological importance of these recombinant noroviruses is still unknown. However, our analyses demonstrated that norovirus has been present throughout the study period regardless of genotypes. Each genotype appeared at times regardless of the low prevalence. The norovirus GII genotypes observed in this study have been circulating globally. The GII.13[P16] that emerged in this study in East Java, Indonesia has become common in places such as India and Europe, suggesting that the minor genotype is epidemiologically important (Hoa-Tran et al., 2015).

In conclusion, we observed that norovirus genotypes circulating in Indonesia were genetically diverse and mostly related to those in Asia and Europe. Since rotavirus vaccines are going to be included in the national immunization program in Indonesia, norovirus rather than rotavirus is expected to become a predominant pathogen in viral diarrhea, and the prevalence and genotypes of norovirus should therefore be continually monitored. The results reported herein demonstrate that noroviruses are important agents responsible for AGE in Indonesian children, and they highlight the importance of continued surveillance.

\section{Declaration of Competing Interest}

The authors declare that they have no conflict of interest.

\section{Acknowledgements}

This research was supported by the Japan Initiative for Global Research Network on Infectious Diseases (J-GRID) from Ministry of Education, Culture, Sport, Science \& Technology in Japan, Japan 


\section{Agency for Medical Research and Development (AMED: 20wm0125009), and the Ministry of Research, Technology and Higher Education (RISTEKDIKTI) of Indonesia.}

\section{References}

Ahmed, S.M., Hall, A.J., Robinson, A.E., Verhoef, L., Premkumar, P., Parashar, U.D., Koopmans, M., Lopman, B.A., 2014. Global prevalence of norovirus in cases of gastroenteritis: a systematic review and meta-analysis. Lancet Infect. Dis. 14, 725-730.

Ao, Y., Wang, J., Ling, H., He, Y., Dong, X., Wang, X., Peng, J., Zhang, H., Jin, M., Duan, Z., 2017. Norovirus GII.P16/GII.2-associated gastroenteritis, China, 2016. Emerg. Infect. Dis. 23, 1172-1175.

Athiyyah, A.F., Utsumi, T., Wahyuni, R.M., Dinana, Z., Yamani, L.N., Soetjipto Sudarmo, S.M., Ranuh, R.G., Darma, A., Juniastuti Raharjo, D., Matsui, C., Deng, L. Abe, T., Doan, Y.H., Fujii, Y., Shimizu, H., Katayama, K., Lusida, M.I., Shoji, I., 2019. Molecular epidemiology and clinical features of rotavirus infection among pediatric patients in East Java, Indonesia during 2015-2018: dynamic changes in rotavirus genotypes from equine-like G3 to typical human G1/G3. Front. Microbiol. 10, 940.

Athiyyah, A.F., Shigemura, K., Kitagawa, K., Agustina, N., Darma, A., Ranuh, R., Raharjo, D., Shirakawa, T., Fujisawa, M., Sudarmo, S.M., 2020. Clinical manifestation of norovirus infection in children aged less than five years old admitted with acute diarrhea in Surabaya, Indonesia: a cross-sectional study. F1000Research 8, 2130.

Bányai, K., Estes, M.K., Martella, V., Parashar, U.D., 2018. Viral gastroenteritis. Lancet 392, 175-186.

Barreira, D.M.P.G., Fumian, T.M., Tonini, M.A.L., Volpini, L.P.B., Santos, R.P., Ribeiro, A. L.C., Leite, J.P.G., Souza, M.T.B.M.E., Brasil, P., da Cunha, D.C., Miagostovich, M.P., Spano, L.C., 2017. Detection and molecular characterization of the novel recombinant norovirus GII.P16-GII.4 Sydney in southeastern Brazil in 2016. PLoS One 12, e0189504.

Bidalot, M., Théry, L., Kaplon, J., De Rougemont, A., Ambert-Balay, K., 2017. Emergence of new recombinant noroviruses GII.P16-GII.4 and GII.P16-GII.2, France, winter 2016 to 2017. Euro. Surveill. 22, 30508.

Bon, F., Ambert-Balay, K., Giraudon, H., Kaplon, J., Le Guyader, S., Pommepuy, M., Gallay, A., Vaillant, V., de Valk, H., Chikhi-Brachet, R., Flahaut, A., Pothier, P., Kohli, E., 2005. Molecular epidemiology of caliciviruses detected in sporadic and outbreak cases of gastroenteritis in France from December 1998 to February 2004. J. Clin. Microbiol. 43, 4659-4664.

Bruggink, L.D., Moselen, J.M., Marshall, J.A., 2016. The comparative molecular epidemiology of GII.P7_GII.6 and GII.P7_GII.7 norovirus outbreaks in Victoria, Australia, 2012-2014. Intervirology 59, 60-65.

Cannon, J.L., Barclay, L., Collins, N.R., Wikswo, M.E., Castro, C.J., Magaña, L.C., Gregoricus, N., Marine, R.L., Chhabra, P., Vinjé, J., 2017. Genetic and epidemiologic trends of norovirus outbreaks in the United States from 2013 to 2016 demonstrated emergence of novel GII.4 recombinant viruses. J. Clin. Microbiol. 57 (e00695-19).

Chhabra, P., de Graaf, M., Parra, G.I., Chan, M.C., Green, K., Martella, V., Wang, Q. White, P.A., Katayama, K., Vennema, H., Koopmans, M.P.G., Vinjé, J., 2019. Updated classification of norovirus genogroups and genotypes. J. Gen. Virol. 100, 1393-1406.

Chuchaona, W., Chansaenroj, J., Wanlapakorn, N., Vongpunsawad, S., Poovorawan, Y., 2019. Recombinant GII.Pe-GII.4 norovirus, Thailand, 2017-2018. Emerg. Infect. Dis. $25,1612-1614$.

Fumian, T.M., da Silva Ribeiro de Andrade, J., Leite, J.P., Miagostovich, M.P., 2016. Norovirus recombinant strains isolated from gastroenteritis outbreaks in Southern Brazil, 2004-2011. PLoS One e0145391, 11.

Giammanco, G.M., De Grazia, S., Bonura, F., Cappa, V., Muli, S.L., Pepe, A., Medici, M.C., Tummolo, F., Calderaro, A., Di Bernardo, F., Dones, P., Morea, A., Loconsole, D. Catella, C., Terio, V., Bànyai, K., Chironna, M., Martella, V., 2017. Norovirus GII.17 as major epidemic strain in Italy, winter 2015-16. Emerg. Infect. Dis. 23, 1206-1208.

Guarines, K.M., Mendes, R.P.G., de Magalhães, J.J.F., Pena, L., 2020. Partial Genome Sequences of Human Norovirus Strains from Northeast Brazil. Microbiol. Resour. Announc. 9 (e01135-19).

Gupta, S., Krishnan, A., Sharma, S., Kumar, P., Aneja, S., Ray, P., 2018. Changing pattern of prevalence, genetic diversity, and mixed infections of viruses associated with acute gastroenteritis in pediatric patients in New Delhi, India. J. Med. Virol. 90, 469-476.

Hakim, M.S., Nirwati, H., Aman, A.T., Soenarto, Y., Pan, Q., 2018. Significance of continuous rotavirus and norovirus surveillance in Indonesia. World J. Pediatr. 14 $4-12$.

Hoa-Tran, T.N., Trainor, E., Nakagomi, T., Cunliffe, N.A., Nakagomi, O., 2013. Molecular epidemiology of noroviruses associated with acute sporadic gastroenteritis in children: global distribution of genogroups, genotypes and GII.4 variants. J. Clin. Virol. 56, 185-193.

Hoa-Tran, T.N., Nakagomi, T., Sano, D., Sherchand, J.B., Pandey, B.D., Cunliffe, N.A., Nakagomi, O., 2015. Molecular epidemiology of noroviruses detected in Nepalese children with acute diarrhea between 2005 and 2011: increase and predominance of minor genotype GII. 13. Infect. Genet. Evol. 30, 27-36.

Jiang, X., Wang, M., Wang, K., Estes, M.K., 1993. Sequence and genomic organization of Norwalk virus. Virology 195, 51-61.

Jin, M., Zhou, Y.K., Xie, H.P., Fu, J.G., He, Y.Q., Zhang, S., Jin, G.H.B., Kong, X.Y., Sun, X.M., Li, H.Y., Zhang, Q., Li, K., Zhang, Y.J., Zhou, D.Q., Xing, W.J., Liao, Q.H., Liu, N., Yu, H.J., Jiang, X., Tan, M., Duan, Z.J., 2016. Characterization of the new
GII.17 norovirus variant that emerged recently as the predominant strain in China. J. Gen. Virol. 97, 2620-2632.

Kageyama, T., Kojima, S., Shinohara, M., Uchida, K., Fukushi, S., Hoshino, F.B., Takeda, N., Katayama, K., 2003. Broadly reactive and highly sensitive assay for Norwalk-like viruses based on real-time quantitative reverse transcription-PCR. J. Clin. Microbiol. 41, 1548-1557.

Khamrin, P., Kumthip, K., Yodmeeklin, A., Supadej, K., Ukarapol, N., Thongprachum, A., Okitsu, S., Hayakawa, S., Ushijima, H., Maneekarn, N., 2016. Molecular characterization of norovirus GII.17 detected in healthy adult, intussusception patient, and acute gastroenteritis children in Thailand. Infect. Genet. Evol. 44, 330-333.

Kobayashi, M., Matsushima, Y., Motoya, T., Sakon, N., Shigemoto, N., OkamotoNakagawa, R., Nishimura, K., Yamashita, Y., Kuroda, M., Saruki, N., Ryo, A., Saraya, T., Morita, Y., Shirabe, K., Ishikawa, M., Takahashi, T., Shinomiya, H., Okabe, N., Nagasawa, K., Suzuki, Y., Katayama, K., Kimura, H., 2016. Molecular evolution of the capsid gene in human norovirus genogroup II. Sci. Rep. 6, 29400.

Kojima, S., Kageyama, T., Fukushi, S., Hoshino, F.B., Shinohara, M., Uchida, K., Natori, K., Takeda, N., Katayama, K., 2002. Genogroup-specific PCR primers for detection of Norwalk-like viruses. J. Virol. Methods 100, 107-114.

Kuang, X., Teng, Z., Zhang, X., 2019. Genotypic prevalence of norovirus GII in gastroenteritis outpatients in Shanghai from 2016 to 2018. Gut Pathog. 11, 40.

Liu, L.T., Kuo, T.Y., Wu, C.Y., Liao, W.T., Hall, A.J., Wu, F.T., 2017. Recombinant GII P16-GII.2 norovirus, Taiwan, 2016. Emerg. Infect. Dis. 23, 1180-1183.

Lole, K.S., Bollinger, R.C., Paranjape, R.S., Gadkari, D., Kulkarn, I.S.S., Novak, N.G., Ingersoll, R., Sheppard, H.W., Ray, S.C., 1999. Full-length human immunodeficiency virus type 1 genomes from subtype C-infected seroconverters in India, with evidence of intersubtype recombination. J. Virol. 73, 152-160.

Louya, V.M., Vouvoungui, C., Koukouikila-Koussounda, F., Veas, F., Kobawila, S.C., Ntoumi, F., 2019. Molecular characterization of norovirus infection responsible for acute diarrhea in Congolese hospitalized children under five years old in Brazzaville, Republic of Congo. Int. J. Infect. Dis. 88, 41-48.

Lu, L., Zhong, H., Xu, M., Su, L., Cao, L., Jia, R., Xu, J., 2019. Genetic diversity and epidemiology of Genogroup II noroviruses in children with acute sporadic gastroenteritis in Shanghai, China, 2012-2017. BMC Infect. Dis. 19, 736.

Martin, D.P., Murrell, B., Golden, M., Khoosal, A., Muhire, B., 2015. RDP4: Detection and analysis of recombination patterns in virus genomes. Virus Evol. 1 (vev003).

Matsushima, Y., Ishikawa, M., Shimizu, T., Komane, A., Kasuo, S., Shinohara, M. Nagasawa, K., Kimura, H., Ryo, A., Okabe, N., Haga, K., Doan, Y.H., Katayama, K., Shimizu, H., 2015. Genetic analyses of GII.17 norovirus strains in diarrheal disease outbreaks from December 2014 to March 2015 in Japan reveal a novel polymerase sequence and amino acid substitutions in the capsid region. Euro. Surveill. 20, 21173.

Matsushima, Y., Mizukoshi, F., Sakon, N., Doan, Y.H., Ueki, Y., Ogawa, Y., Motoya, T., Tsukagoshi, H., Nakamura, N., Shigemoto, N., Yoshitomi, H., OkamotoNakagawa, R., Suzuki, R., Tsutsui, R., Terasoma, F., Takahashi, T., Sadamasu, K., Shimizu, H., Okabe, N., Nagasawa, K., Aso, J., Ishii, H., Kuroda, M., Ryo, A., Katayama, K., Kimura, H., 2019. Evolutionary analysis of the VP1 and RNAdependent RNA polymerase regions of human norovirus GII.P17-GII.17 in 2013-2017. Front. Microbiol. 10, 2189.

Medici, M.C., Morelli, A., Arcangeletti, M.C., Calderaro, A., De Conto, F., Martinelli, M., Abelli, L.A., Dettori, G., Chezzi, C., 2009. An outbreak of norovirus infection in an Italian residential-care facility for the elderly. Clin. Microbiol. Infect. 15, 97-100.

Medici, M.C., Tummolo, F., Martella, V., Giammanco, G.M., De Grazia, S., Arcangeletti, M.C., De Conto, F., Chezzi, C., Calderaro, A., 2014. Novel recombinant GII.P16_GII.13 and GII.P16_GII.3 norovirus strains in Italy. Virus. Res. 188, 142-145.

Motoya, T., Umezawa, M., Saito, A., Goto, K., Doi, I., Fukaya, S., Nagata, N., Ikeda, Y., Okayama, K., Aso, J., Matsushima, Y., Ishioka, T., Ryo, A., Sasaki, N., Katayama, K., Kimura, H., 2019. Variation of human norovirus GII genotypes detected in Ibaraki, Japan, during 2012-2018. Gut. Pathog. 11, 26.

Nagasawa, K., Matsushima, Y., Motoya, T., Mizukoshi, F., Ueki, Y., Sakon, N. Murakami, K., Shimizu, T., Okabe, N., Nagata, N., Shirabe, K., Shinomiya, H., Suzuki, W., Kuroda, M., Sekizuka, T., Ryo, A., Fujita, K., Oishi, K., Katayama, K., Kimura, H., 2018. Phylogeny and immunoreactivity of norovirus GII.P16-GII.2, Japan, winter 2016-17. Emerg. Infect. Dis. 24, 144-148.

Niendorf, S., Jacobsen, S., Faber, M., Eis-Hübinger, A.M., Hofmann, J., Zimmermann, O., Höhne, M., Bock, C.T., 2017. Steep rise in norovirus cases and emergence of a new recombinant strain GII.P16-GII.2, Germany, winter 2016. Euro. Surveill. 22, 30447.

Nirwati, H., Donato, C.M., Mawarti, Y., Mulyani, N.S., Ikram, A., Aman, A.T., Peppelenbosch, M.P., Soenarto, Y., Pan, Q., Hakim, M.S., 2019. Norovirus and rotavirus infections in children less than five years of age hospitalized with acute gastroenteritis in Indonesia. Arch. Virol. 164, 1515-1525.

Parra, G.I., 2019. Emergence of norovirus strains: a tale of two genes. Virus Evol. 5 , vez048.

Polkowska, A., Rönnqvist, M., Lepistö, O., Roivainen, M., Maunula, L., Huusko, S., Toikkanen, S., Rimhanen-Finne, R., 2014. Outbreak of gastroenteritis caused by norovirus GII.4 Sydney variant after a wedding reception at a resort/activity centre, Finland, August 2012. Epidemiol. Infect. 142, 1877-1883.

Siafakas, N., Zerva, L., Hatzaki, D., Lebessi, E., Chronopoulou, G., Paraskakis, I., Pournaras, S., 2018. Molecular epidemiology of noroviruses in children in South Greece, 2013-2015. J. Med. Virol. 90, 1703-1711.

Sudarmo, S.M., Shigemura, K., Athiyyah, A.F., Osawa, K., Wardana, O.P., Darma, A., Ranuh, R., Raharjo, D., Arakawa, S., Fujisawa, M., Shirakawa, T., 2015. Genotyping and clinical factors in pediatric diarrhea caused by rotaviruses: one-year surveillance in Surabaya, Indonesia. Gut Pathog. 7 (3).

Tamura, K., Dudley, J., Nei, M., Kumar, S., 2007. MEGA4: molecular evolutionary genetics analysis (MEGA) software version 4. 0. Mol. Biol. Evol. 24, 1596-1599. 
Thongprachum, A., Takanashi, S., Kalesaran, A.F., Okitsu, S., Mizuguchi, M., Hayakawa, S., Ushijima, H., 2015. Four-year study of viruses that cause diarrhea in Japanese pediatric outpatients. J. Med. Virol. 87, 1141-1148.

Tse, E.H.Y., Zhang, L.Y., Lau, S.L., Chan, M.C., 2020. Genome sequence of a human norovirus GII.4 Hong Kong[P31] variant in Hong Kong, China. Microbiol. Resour. Announc. 9 (e01391-19).

Utsumi, T., Lusida, M.I., Dinana, Z., Wahyuni, R.M., Yamani, L.N., Soetjipto, Juniastuti, Matsui, C., Deng, L., Abe, T., Doan, Y.H., Fujii, Y., Kimura, H., Katayama, K., Shoji, I., 2017. Occurrence of norovirus infection in an asymptomatic population in Indonesia. Infect. Genet. Evol. 55, 1-7.

van Beek, J., de Graaf, M., Al-Hello, H., Allen, D.J., Ambert-Balay, K., Botteldoorn, N., Brytting, M., Buesa, J., Cabrerizo, M., Chan, M., Cloak, F., Di Bartolo, I., Guix, S., Hewitt, J., Iritani, N., Jin, M., Johne, R., Lederer, I., Mans, J., Martella, V., Maunula, L., McAllister, G., Niendorf, S., Niesters, H.G., Podkolzin, A.T., PoljsakPrijatelj, M., Rasmussen, L.D., Reuter, G., Tuite, G., Kroneman, A., Vennema, H., Koopmans, M.P.G., NoroNet, 2018. Molecular surveillance of norovirus, 2005-16: an epidemiological analysis of data collected from the NoroNet network. Lancet Infect. Dis. 18, 545-553.

Vinjé, J., 2015. Advances in laboratory methods for detection and typing of norovirus. J. Clin. Microbiol. 53, 373-381.

Wang, X., Wei, Z., Guo, J., Cai, J., Chang, H., Ge, Y., Zeng, M., 2019. Norovirus activity and genotypes in sporadic acute diarrhea in children in Shanghai During 2014-2018. Pediatr. Infect. Dis. J. 38, 1085-1089.
Wangchuk, S., Matsumoto, T., Iha, H., Ahmed, K., 2017. Surveillance of norovirus among children with diarrhea in four major hospitals in Bhutan: replacement of GII.21 by GII.3 as a dominant genotype. PLoS One 12, e0184826.

Wu, X., Han, J., Chen, L., Xu, D., Shen, Y., Zha, Y., Zhu, X., Ji, L., 2015. Prevalence and genetic diversity of noroviruses in adults with acute gastroenteritis in Huzhou, China, 2013-2014. Arch. Virol. 160, 1705-1713.

Wu, C.Y., Chi, H., Liu, C.C., Huang, Y.C., Huang, Y.C., Lin, H.C., Ho, Y.H., Huang, L.M., Huang, C.Y., Shih, S.M., Wu, F.T., Mu, J.J., Hsiung, C.A., 2020. Clinical characteristics and risk factors for children with norovirus gastroenteritis in Taiwan. J. Microbiol. Immunol. Infect. 11 (S1684-1182).

Wulandari, P.S., Juniastuti Wahyuni, R.M., Amin, M., Yamani, L.N., Matondang, M.Q.Y., Dinana, Z., Soetjipto Utsumi, T., Shoji, I., Lusida, M.I., 2020. Predominance of norovirus GI.4 from children with acute gastroenteritis in Jambi, Indonesia, 2019. J. Med. Virol. 92, 3165-3172.

Xue, L., Cai, W., Gao, J., Zhang, L., Dong, R., Li, Y., Wu, H., Chen, M., Zhang, J., Wang, J., $\mathrm{Wu}, \mathrm{Q} ., 2$ 2019. The resurgence of the norovirus GII.4 variant associated with sporadic gastroenteritis in the post-GII.17 period in South China, 2015 to 2017. BMC Infect. Dis. 19, 696.

Zheng, D.P., Widdowson, M.A., Glass, R.I., Vinjé, J., 2010. Molecular epidemiology of genogroup II-genotype 4 noroviruses in the United States between 1994 and 2006. J. Clin. Microbiol. 48, 168-177. 